\title{
Quantum interference and the giant Hall effect in percolating systems
}

\author{
Chuncheng Wan, ${ }^{1,2}$ and Ping Sheng ${ }^{1}$ \\ ${ }^{1}$ Department of Physics and the Institute of Nano Science and Technology, The Hong Kong University of Science and Technology, \\ Clear Water Bay, Kowloon, Hong Kong, China \\ ${ }^{2}$ The Chemistry Department, University of Toronto, Toronto, Ontario, Canada
}

(Received 13 April 2001; revised manuscript received 25 March 2002; published 5 August 2002)

\begin{abstract}
We show that in metallic percolating networks the wave nature of the charge carriers can significantly modify the classical picture of the Hall effect, especially in the metal concentration range $x \approx x_{q}$, where $x$ denotes the metal volume, and $x_{q}$ the quantum-percolation threshold. Calculations based on the model of local quantum interference effect are shown to give a consistent and quantitative account of the recent experimental findings on the (ordinary) giant Hall effect.
\end{abstract}

DOI: 10.1103/PhysRevB.66.075309

PACS number(s): 72.20.My, 72.15.Rn, 73.50.Jt

Percolation is a geometric concept basic to the study of physical properties of inhomogeneous materials. In metalinsulator composites, the assumption that the charge carriers are classical point particles leads directly to the prediction of a conductivity transition at the geometric percolation threshold $x_{c}$, below which the metallic component can no longer form an infinite network. Consideration of the wave nature of the physical charge carriers (electrons), however, leads to a somewhat different picture at temperature $T=0$, when inelastic scattering is absent. That is, multiple scattering of the electronic waves induced by the random percolating geometry of the conducting channels inevitably localizes the electronic wave functions at a metal concentration $x_{q}>x_{c}\left[x_{q}\right.$ $=1$ in one-dimensional (1D) and 2D samples], denoted the quantum mobility edge. As a result, for $x_{c}<x<x_{q}$ the predictions of the classical- and the quantum-percolation models are at odds with each other: Whereas the metallic networks are connected and therefore the classical-percolation model predicts metallic behavior, the quantum-percolation model predicts a nonmetallic behavior. ${ }^{1}$ Indeed, both types of behavior have been observed in thin (2D) metallic films. Whereas the nonmetallic temperature dependence observed at low temperatures is widely accepted as due to the weaklocalization effect arising from quantum wave interferences, ${ }^{2,3}$ at higher temperatures inelastic scattering is seen to suppress wave effects and restore the metallic behavior predicted by classical percolation. Beyond the 2D samples and the weak localization at low temperatures, however, consideration of quantum wave interference effects has been lacking for Hall effect in 3D samples in the concentration range $x \sim x_{q}$, where wave effects could be potentially significant. In view of recent experimental findings on the giant Hall effect (GHE), ${ }^{4-7}$ a theory of Hall effect that takes into consideration the wave effects would be especially called for.

As a basic material constant, the Hall coefficient is generally indicative of the density and sign of the charge carriers. Thus, in granular metals, as the metal concentration decreases, the lower carrier density is expected to yield an enhanced Hall coefficient that peaks at the percolation threshold with a factor of $\sim 30$ for $\sim 1 \mu \mathrm{m}$ thick films. ${ }^{4}$ Recently, however, it was found that in the magnetic $(\mathrm{NiFe}) \mathrm{SiO}_{2}$ and $\mathrm{FeSiO}_{2}$ granular films, ${ }^{4-7}$ the extraordinary
Hall coefficient was enhanced by a factor of $10^{4}$ when the metal volume fraction is close to the classical-percolation threshold of $x=0.53$. Even after magnetic saturation, the ordinary Hall coefficient was still observed to increase by almost three orders of magnitude, ${ }^{6}$ suggesting that a magnetism-independent mechanism could be operative. The origin of this ordinary GHE was investigated by using nonmagnetic $\mathrm{CuSiO}_{2}$ granular films, with the result that not only the maximum enhancement magnitude coincided with the ordinary GHE observed in magnetic composites, but more importantly the mechanism was also found to be correlated with quantum interference. ${ }^{8}$ In particular, the maximum enhancement was found at the quantum-percolation threshold, determined experimentally by the Ioffe-Regel criterion of $k l \sim 1,{ }^{3}$ where $k$ is the electronic wave vector and $l$ the mean free path. The enhancement was also found to vanish when the feature size of the granular film was increased (through annealing) to being comparable to the electron dephasing length. It is the purpose of this work to present a theoretical model of GHE based on the mechanism of local quantum interference. We show that in those cases where the microstructural feature size $\xi$ is smaller then the temperaturedependent dephasing length $L_{\phi}(T)=\sqrt{l_{\text {ine }} l / 3}{ }^{3}$ where $l_{\text {ine }}$ denotes the inelastic scattering length and $l$ the elastic mean free path, there can be significant quantum interference effects even beyond the low-temperature regime. In particular, calculations based on the 3D quantum-percolation model predict a drastically different physical picture for the Hall effect in the range $x \sim x_{q}$, even when $L_{\phi}(T)$ is only on the order of a few $\xi$ 's. Numerical results based on our picture are shown to give a good account for the GHE found in nonmagnetic $\mathrm{CuSiO}_{2}$ granular composites. ${ }^{8}$

Consider a 3D sample of disordered metal-insulator composite in the percolating regime, characterized by an average geometric feature size $\xi$ defined by the small insulating (or metallic) particles and their separations, which form the conducting channels. At finite temperatures, the existence of a dephasing length $L_{\phi}(T)$ means that the wave effects are negligible on a scale larger then $L_{\phi}(T)$. Hence, the overall transport characteristics of the sample may be treated as a classical network problem in which each element of the network has the properties calculated on the scale of $L_{\phi}(T)$, within which the quantum wave interference effects have to be fully taken into account. The importance of such quantum effects 
may be judged by comparing $\xi$ with $L_{\phi}(T)$. If $L_{\phi}(T)<\xi$, wave dephasing occurs within a single feature size, i.e., in either the metal or the insulator. The wave effects should be minimal in that case, and the classical-percolation picture prevails. The interesting case occurs when $L_{\phi}(T)>\xi$. Being sensitive to the details of the microstructure, quantum wave interference can lead to diverse effective properties (at different spatial locations in the sample) differing qualitatively from those of either the metal or the insulator component. As $\xi$ can be on the order of $1 \mathrm{~nm}$, it follows that the effects of local quantum interference may persist even beyond the lowtemperature regime. This sensitivity on the feature size was indeed demonstrated experimentally through sample annealing, which showed that as the feature size increases to the level comparable to or larger than the measured dephasing length, the GHE disappears. ${ }^{8}$

Below we focus on the calculation of the Hall effect in 3D percolating networks based on the above picture. In the composition range $x \sim x_{q}$ the local Hall coefficient, calculated on the basis of $L_{\phi}(T) \approx a$ few $\xi$ 's, is shown to be dominated by wave scattering and interference, and can be of either positive or negative sign with roughly equal probability. The giant Hall effect is a direct result of this local behavior when properly averaged over the whole sample. We also show that quantum interference can give rise to a distribution of local longitudinal conductances, which can directly affect the conductivity behavior as $x$ approaches $x_{c}$. While the purpose of this work is not to investigate the detailed behavior of longitudinal conductances in the 3D quantum percolation model, we nevertheless wish to use longitudinal conductances as the variable to monitor Hall coefficient variation. This is desirable, because the correlation between the two should reflect the intrinsic physics that may be directly compared to experiment.

For the calculation of effective properties on the scale of $L_{\phi}$, we use the $3 \mathrm{D}$ quantum-percolation model defined on a finite $\left(L_{\phi} \times L_{\phi} \times L_{\phi}\right)$ simple cubic lattice. Here the lattice constant may be regarded as a measure of the geometric feature size (grain size) $\xi$. The Hamiltonian of the model is defined by ${ }^{1}$

$$
\begin{aligned}
H= & -\sum_{\langle m n\rangle} t_{m n}\left[\exp \left(i A_{m n} / \phi_{0}\right) a_{m}^{\dagger} a_{n}\right. \\
& \left.+\exp \left(-i A_{m n} / \phi_{0}\right) a_{n}^{\dagger} a_{m}\right]+\sum_{m} w_{m} a_{m}^{\dagger} a_{m},
\end{aligned}
$$

where $a_{m}^{\dagger}$ creates a state at site $m=\{i, j, k\}$, where $i, j, k$ are the indices along the three orthogonal directions, and $\phi_{0}$ $=h c / e$ is the unit quantum flux. In the following we assume $t_{m n}=t$, and $\langle m n\rangle$ means nearest-neighbor pairs. Since the electron is forbidden from entering the insulating particles, we have $w_{m}=\infty$ if $m$ is an insulating site, and $w_{m}=0$ if $m$ is metallic. $A_{m n}$ is the gauge field induced by a uniform magnetic field $B$ in the $z$ direction, perpendicular to the applied electric field, chosen as $A_{m, m+\{0,0,1\}}=A_{m, m+\{0,1,0\}}=0$, and $A_{m, m+\{1,0,0\}}=-B b_{y} b_{z} j$, where $b=b_{x}, b_{y}, b_{z}$ is the bond length in the three directions, reflecting, in general, the geometrical feature size.

Within the dephasing length $L_{\phi}$, we need to consider the complete quantum wave function as a result of the Hamil- tonian given by Eq. (1). For this purpose, we perform exactnumerical diagonalization of the Hamiltonian (1) on a $L_{\phi}$ $\times L_{\phi} \times L_{\phi}$ lattice, with generalized periodic boundary conditions. ${ }^{9}$ It is noted, however, that the final result is not sensitive to the actual boundary condition due to the random geometry of the lattice. In accordance with the linearresponse theory, the Hall conductance $G_{H}$ for the coherent region can be calculated by the Kubo formula: ${ }^{10}$

$$
G_{H}=\frac{i \hbar e^{2}}{L_{\phi}^{2}} \sum_{\mu} \frac{\left\langle\Psi\left|V_{x}\right| \mu\right\rangle\left\langle\mu\left|V_{y}\right| \Psi\right\rangle-(\text { H.c. })}{\left(E_{\mu}-E_{\Psi}\right)^{2}} .
$$

where $\Psi$ denotes the initial (ground) state, the sum is over all excited state denoted by $\mu$, obtained from numerical diagonalization, and $L_{\phi}$ is the system size. $V_{x}$ and $V_{y}$ are velocity operators defined as

$$
\begin{gathered}
V_{x}=\frac{i b t}{\hbar} \sum_{m} a_{m+\{1,0,0\}}^{\dagger} a_{m} \exp \left(i B b_{y} b_{z} \frac{2 \pi j}{\phi_{0}}\right) \\
-a_{m}^{+} a_{m+\{1,0,0\}} \exp \left(-i B b_{y} b_{z} \frac{2 \pi j}{\phi_{0}}\right), \\
V_{y}=\frac{i b t}{\hbar} \sum_{m} a_{m+\{0,1,0\}}^{\dagger} a_{m}-a_{m}^{\dagger} a_{m+\{0,1,0\}},
\end{gathered}
$$

where the summations are over all sites within a given region of coherence. It should be noted that $G_{H}$ is sensitive to the actual geometry of the percolating network within each region. While the metal average density is maintained as an overall constant, the actual composition and geometry of the small regions can vary randomly from one to the next. As a result, there is necessarily a distribution of $G_{H}$. We have carried out numerical evaluations of $G_{H}$ for randomly generated finite samples with the Fermi energy $E_{f}$ near the band center. We have found that other than a scaling factor, the function $G_{H}$ is independent of the choice of $E_{f}$. Shown in Fig. 1(a) are the distributions of $\sigma_{H}=G_{H} / L_{\phi}$, the averaged Hall conductivity, at two different metal concentrations obtained from 5000 randomly generated samples with $L_{\phi}$ equal to 10 lattice constants. In the calculations we have used the value of $B b_{y} b_{z} / \phi_{0} \sim 0.5$. We have also monitored the magnetic-field dependence of $\sigma_{H}$ (see below) from $B=0$ to $B b_{y} b_{z} / \phi_{0} \sim 0.5$. It is interesting to note that for $x=0.35$, which is below $x_{q}$, but above $x_{c}$, the distribution is fairly symmetrical about zero; whereas for the case of $x=0.55$ $>x_{q}$, the center of the distribution notably shifts to the negative side, so that the mean corresponds with the sign of the charge carrier. In other words, $\sigma_{H}$ can be either negative or positive due to the quantum interference effect. Thus local quantum interference can lead to very different local properties in percolating systems.

For the longitudinal conductance $G$, it is usually more convenient to treat the problem with the Landauer-Buttiker approach, ${ }^{11}$ which is theoretically similar to the Kubo formula. We have performed numerical evaluations of $G$ for the same 5000 randomly generated samples as that used in the $G_{H}$ calculation. The calculated distributions are shown in Fig. 1(b) for two metal concentrations. It is striking that the distributions display peaks at small values of the conduc- 

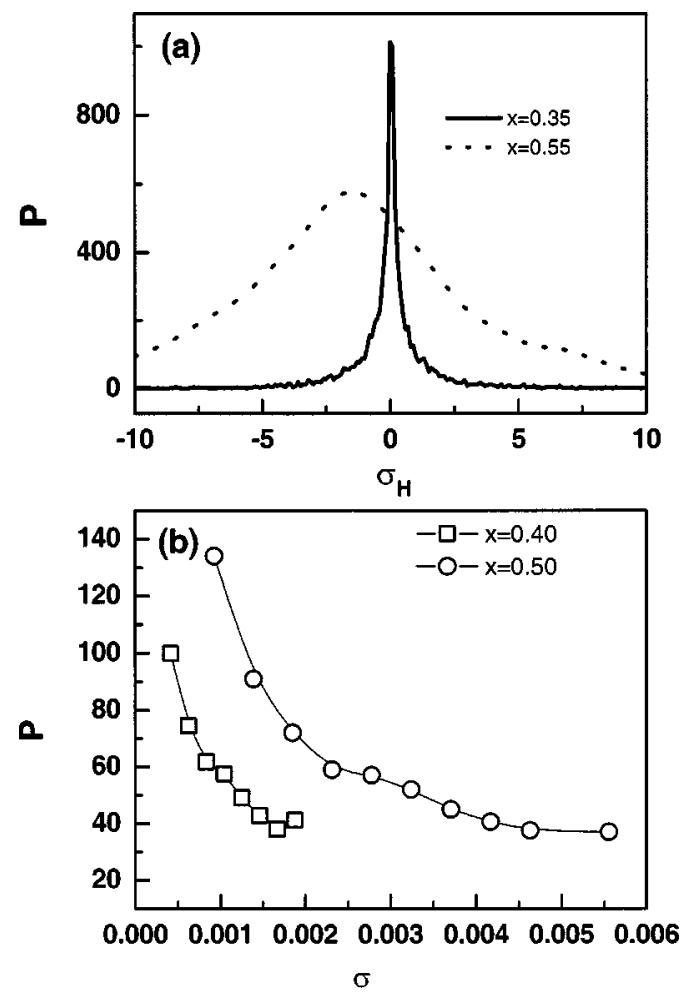

FIG. 1. (a) Calculated distributions of local Hall conductivity $\sigma_{H}=G_{H} / L_{\phi}$ at two metal concentrations. The distribution is noted to be nearly symmetrical for $x=0.35$. (b) Calculated distributions of local longitudinal conductivity $\sigma=G / L_{\phi}$ at two metal concentrations. Due to the localizing effect of wave interference, the distributions display a preponderance of small conductivities.

tance. Here we propose that local quantum interference effect can be one mechanism for generating nearly singular distributions of $\sigma$. Our calculations of $\sigma$ and $\sigma_{H}$ suggest that each local region of the macroscopic sample is characterized by a conductivity tensor $\widetilde{\sigma}$, with $\sigma=\sigma_{x x}=\sigma_{y y}=\sigma_{z z}$ for the diagonal components, $\sigma_{x y}=-\sigma_{y x}=\sigma_{H}$, and $\sigma_{x z}=\sigma_{z x}=0$. To calculate the macroscopic effective conductivity tensor, we use the conditions of $\boldsymbol{\nabla} \times \mathbf{E}=0$ and $\boldsymbol{\nabla} \cdot \mathbf{j}=0$, where the current density $\mathbf{j}=\widetilde{\sigma} \mathbf{E}$. These two conditions, equivalent to the Kircchoff equations for discrete electrical networks, can be combined to yield $\boldsymbol{\nabla} \cdot \widetilde{\sigma} \boldsymbol{\nabla} \varphi=0$, where $\varphi$ denotes the electrical potential. We have numerically solved the above equation on a discretized $10 \times 10 \times 10$ lattice, where each lattice site has to be understood as consisting of a region of the size $L_{\phi} \times L_{\phi} \times L_{\phi}$. The condition of unit voltage difference along the $x$ direction and periodic in the other directions is imposed, and the values of the conductivity tensor components, assigned to each node of the classical electrical network, are randomly selected from those calculated previously (from randomly generated configurations). We denote the Hall conductivity and Hall resistivity so calculated by $\bar{\sigma}_{H}$ and $\bar{\rho}_{H}$, respectively. To examine the finite-size effect associated with the classical network, we have made comparative calculations on a $8 \times 8 \times 8$ lattice. It can be seen in Fig. 2 that the two sizes yield nearly identical results, and both are very close to the mean value $\left\langle\sigma_{H}\right\rangle$ of the $\sigma_{H}$ distribution, shown as the solid line in Fig. 2. This is plausible, since in our case

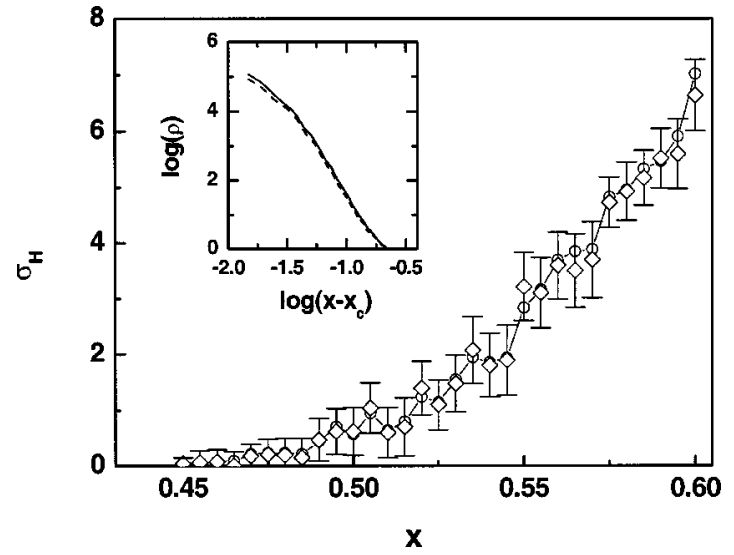

FIG. 2. Hall conductance obtained from networks of two different sizes: $10 \times 10 \times 10$ (circles), $8 \times 8 \times 8$ (diamonds). The solid line gives the mean value of the distribution. The inset shows a log-log plot of the longitudinal resistance as a function of metal concentration for two sizes: $10 \times 10 \times 10$ (solid line) and $8 \times 8 \times 8$ (dashed line).

the Hall coefficient is found to vary as $R_{H}=\bar{\rho}_{H} / B$ $\cong \bar{\sigma}_{H}^{-1} B^{-1},{ }^{12}$ which is appropriate in the "high-field" limit. We have determined this by carrying out calculations on the $B$ variation of $\bar{\sigma}_{H}$, and found that when $B b_{y} b_{z} / \phi_{0} \sim 0.5, \bar{\sigma}_{H}$ varies as $1 / B .{ }^{12}$ Since $R_{H}$ is usually in the form of $1 / n e c$, where $n$ is the charge carrier density, $e$ the electronic charge, and $c$ the speed of light. Our result shows that $\bar{\sigma}_{H} \cong\left\langle\sigma_{H}\right\rangle$ $=\int \sigma_{H} P\left(\sigma_{H}\right) d \sigma_{H}$, which translates into $\bar{\sigma}_{H} \propto\langle n\rangle e c$, or $\bar{R}_{H}$ $\cong 1 /\langle n\rangle$. The fact that the $\sigma_{H}$ distribution is nearly symmetrical around zero (so that $\langle n\rangle \sim 0$ ) thus directly translates into the giant Hall effect. Also shown in the inset of Fig. 2 is the $\log -\log$ plot of the longitudinal resistance as a function of metal concentration, obtained from the networks of the two sizes. It is again seen that the finite-size effect in this case is also minimal. In Fig. 3(a) the effective Hall coefficient $\bar{R}_{H}$ is plotted as a function of $x-x_{c}$, with $x_{c} \cong 0.31$. There is a clear three-segment behavior, marked by a sudden increase in the Hall coefficient in the vicinity of the $x_{q}$, followed by a plateau. Mathematically, this is due to the steady shift of $\left\langle\sigma_{H}\right\rangle$ towards smaller values as the metal concentration decreases. It should be noted that due to the finite size of the samples involved in the calculations $\left(L_{\phi} \times L_{\phi} \times L_{\phi}\right)$, the peak position of $R_{H}$ cannot correspond exactly with $x_{q}$, which is determined at $T=0$ or the limit $L_{\phi} \rightarrow \infty$. Rather, the peak occurs at a value $x<x_{q}$ where the localization length is comparable to $L_{\phi}$. Also, since the present mechanism of the giant Hall effect is based on the near-complete cancellation of positive and negative Hall conductances [due to the symmetry of $P\left(\sigma_{H}\right)$ ], a direct implication is that there should be significant fluctuations in the overall value of $R_{H}$ near its peak. This has indeed been observed experimentally near the highest values of the Hall coefficient, as samples with almost the identical metal concentration can yield Hall coefficients that differ by a factor of 2 or more.

In the present model, temperature dependence enters through the variation of the dephasing length. We have carried out extensive calculations by using $L_{\phi}(T)=3$ (high 

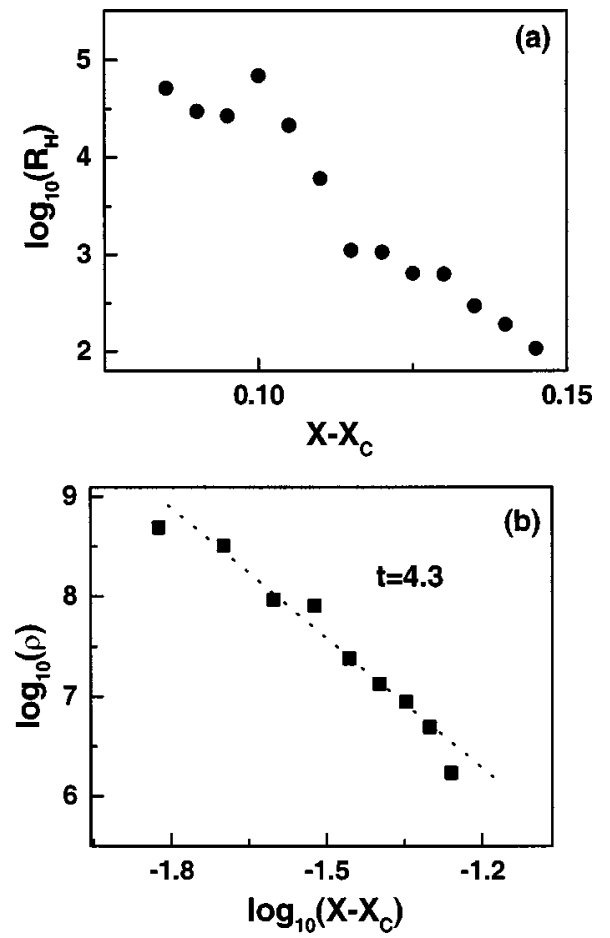

FIG. 3. (a) Effective Hall coefficient plotted as a function of $x$ $-x_{c}$. Note the fact that the peak occurs $\sim x_{q}>x_{c}$. (b) $\ln \rho$ plotted as a function of $\ln \left(x-x_{c}\right)$. The slope of the dashed line gives a slope value of 4.3, observed in $\mathrm{CuSiO}_{2}$ granular composites.

temperatures) up to $L_{\phi}(T)=10$ (low temperatures), and found that the Hall effect is essentially temperature independent within this range. This is also in good correspondence with what has been observed experimentally in granular $\mathrm{CuSiO}_{2} \cdot{ }^{8} \mathrm{~A}$ simple explanation is that the overall Hall effect is sensitive only to the mean of $P\left(\sigma_{H}\right)$. Therefore, while the distribution itself can vary as $L_{\phi}(T)$ varies, its mean may nevertheless remains similar.

In Fig. 3(b) $\ln \rho$ is plotted versus $\ln \left(x-x_{c}\right)$. The best fit gives a slope of 4.3 , which is larger than the conventional value of 2. This slope value is noted to be in excellent agreement with that observed in the $\mathrm{CuSiO}_{2}$ granular system. ${ }^{8}$ However, it should also be noted that the segment further away from $x_{c}$ has a slope larger than 4.3, whereas the segment closer to $x_{c}$ has a slope smaller than 4.3. Such behavior reflects the variation of the local longitudinal conductance distribution as a function of metal concentration, shown in Fig. 1(b). Hence the interpretation of this slope as the critical exponent is in doubt. We would like to note that exponent values greater than 2 have been widely reported in the literature. ${ }^{13}$ Our calculations are suggestive of quantum in-

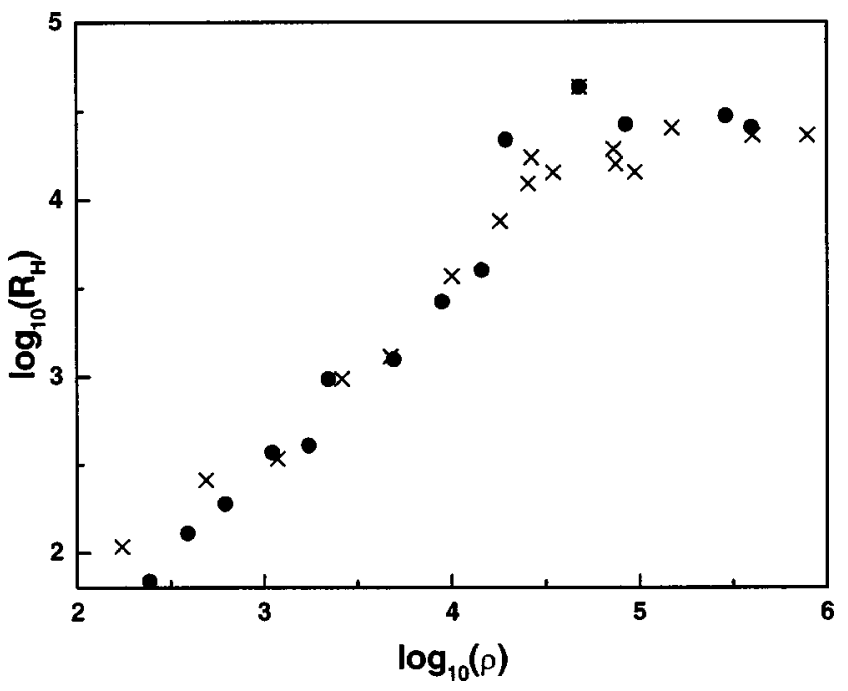

FIG. 4. $\ln R_{H}$ plotted versus $\ln \rho$. Theory points are indicated by solid circles. Experimental data are shown by crosses. The theory and experimental data are normalized at one point-the peak value of the Hall coefficient and its associated longitudinal resistivity. Semiquantitative agreement is seen between the theoretical predictions and the measured data, taken from Ref. 8.

terference as a mechanism for such behavior, but also raise some doubts on their interpretation as critical exponent values. Our results are not yet definitive on this point.

In Fig. 4 we cross-plot $R_{H}$ against $\rho$ on the log-log scale. Due to the orders of magnitude variations in both $R_{H}$ and $\rho$, this offers the best quantitative comparison between theory and experiment. Such a plot also eliminates the need to determine $x_{c}$ or $x_{q}$. Experimental results, denoted by crosses, are plotted on the same graph by normalizing the point of experimental peak Hall coefficient with the same theory point. The theoretically predicted characteristics are noted to give not only the general overall trend of measured data, but are also in semiquantitative agreement with the magnitude of the enhancement, $\sim 1000$ times, in the Hall coefficient. ${ }^{8}$

In summary, we have presented a theory on the local quantum interference effect in the electronic transport in granular metal-insulator composites. This quantum effect occurs at finite temperatures with finite dephasing length. We show that the quantum interference effect can considerably alter both the Hall and longitudinal conductivity behavior. In particular, it offers a consistent quantitative explanation of the giant Hall effect in the nonmagnetic granular $\mathrm{CuSiO}_{2}$.

This work was supported by RGC Grant No. HKUST612/ 95P. We wish to thank X. X. Zhang for many helpful discussions about the experimental data and their implications.
${ }^{1}$ S. Kirkpatrick, Phys. Rev. B 6, 3598 (1972); C. M. Soukoulis, E. N. Economou, and G. S. Grest, ibid. 36, 8649 (1987). Since $x_{c}$ and $x_{q}$ are nonuniversal values, they can vary from system to system. In $\mathrm{CuSiO}_{2}$ granular metal films, $x_{c} \approx 0.43$ and $x_{q}$ $\approx 0.51$. In lattice-percolation models, $x_{c} \approx 0.31$ and $x_{q}$ $\approx 0.37-0.4$.
${ }^{2}$ G. J. Dolan and D. D. Osheroff, Phys. Rev. Lett. 43, 721 (1979).

${ }^{3} \mathrm{P}$. Sheng, Introduction to Wave Scattering, Localization, and Mesoscopic Phenomena (Academic, Boston, 1995), p. 284.

${ }^{4}$ X. N. Jing et al., Phys. Rev. B 53, 14032 (1996).

${ }^{5}$ A. B. Pakhomov, X. Yan, and B. Zhao, Appl. Phys. Lett. 67, 3497 (1995). 
${ }^{6}$ A. B. Pakhomov and X. Yan, Solid State Commun. 99, 139 (1996); Physica A 229, 402 (1996); A. B. Pakhomov et al., ibid. 241, 344 (1997).

${ }^{7}$ B. A. Zronzon et al., Phys. Status Solidi B 205, 151 (1998); JETP Lett. 70, 90 (1999).

${ }^{8}$ X. X. Zhang, C. Wan, H. Liu, Z. Q. Li, Ping Sheng, and J. J. Lin, Phys. Rev. Lett. 86, 5562 (2001).

${ }^{9}$ Q. Niu, D. Thouless, and Y. S. Wu, Phys. Rev. B 31, 3372 (1985).

${ }^{10}$ R. Kubo, Can. J. Phys. 34, 1274 (1956); A. D. Greenwood, Proc. Phys. Soc. Jpn. 71, 585 (1958).
${ }^{11}$ M. Buttiker, Y. Imry, R. Landauer, and S. Pinhas, Phys. Rev. B 31, 6207 (1985).

${ }^{12}$ Classically, $\bar{\sigma}_{H} \sim \omega_{c} \tau \gamma \sigma$, and $\bar{\sigma}_{H} \sim \omega_{c} \tau / \sigma$, with the latter obtained from the inversion of the $2 \times 2$ conductivity matrix. Here $\omega_{c}=e B / m c, \gamma=\left(1+\omega_{c}^{2} \tau^{2}\right)^{-1}$, and $\tau$ is the electron-scattering time. Thus in the small $B$ limit, $\bar{\sigma}_{H} \sim B$. But in the large $B$ limit, $\bar{\sigma}_{H} \sim 1 / B$, and $\bar{\rho}_{H} \cong 1 / \bar{\sigma}_{H}$. Therefore $R_{H}=\bar{\rho}_{H} / B \cong \bar{\sigma}_{H}^{-1} B^{-1}$.

${ }^{13}$ D. S. McLachlan, K. Cai, C. Chiteme, and W. D. Heiss, Physica B 279, 66 (2000). 